\title{
A Modelagem Computacional no Ensino de Física: Um Estudo Exploratório sobre o Oscilador Harmônico Simples
}

Carlos Henrique Santos Verbeno ch-kb@hotmail.com

0000-0002-7009-4589

Universidade Federal do Espírito Santo, Vitória, Espírito Santo, Brasil.

Rodrigo Marques Almeida da Silva

rodrigomas@gmail.com

Pontifícia Universidade Católica do Rio de Janeiro, Rio de Janeiro, Brasil.

\section{Rômulo Maziero}

maziero.ifes@gmail.com

0000-0002-5739-1941

Espírito Santo, São

Mateus, Espírito Santo, Brasil.

\section{Thiéberson da Silva Gomes} thieberson@gmail.com

0000-0002-8432-6061

Universidade Federal do Espírito Santo,

Vitória, Espírito Santo, Brasil.

Laércio Ferracioli

$\frac{\text { laercio.ufes@gmail.com }}{\text { 0000-0002-8119-3232 }}$

Universidade Federal do Espírito Santo,

Vitória, Espírito Santo, Brasil.

\begin{abstract}
RESUMO
Este trabalho investigou a integração de um módulo educacional com base em atividades de modelagem computacional no estudo de um tópico específico de Ciências: o Oscilador Harmônico Simples (OHS). O estudo consistiu do planejamento, implementação, teste e avaliação do módulo educacional. Os instrumentos de coleta de dados foram divididos em duas partes: as atividades de modelagem e a planilha de avaliação do módulo educacional. $\mathrm{O}$ teste foi realizado com professores de Física do Ensino Médio cursando pós-graduação na Universidade Federal do Espírito Santo. Os dados da avaliação do módulo educacional demonstraram a adequação ao objetivo a que se propôs, sendo que, foram implementadas as modificações sugeridas para utilização futura. Por outro lado, os resultados das atividades de modelagem mostraram que os professores puderam confrontar o entendimento prévio sobre os conceitos abordados com os resultados da simulação e, em alguns casos, reforçar suas concepções alternativas e em outros evoluí-las ao conhecimento científico.
\end{abstract}

PALAVRAS-CHAVE: Ensino de Física. Módulo Educacional. 


\section{INTRODUÇÃO}

A questão da utilização da tecnologia no contexto educacional é um tema atual e gerador de fóruns de debate como simpósios e conferências tanto a nível nacional quanto internacional. $\mathrm{O}$ avanço e barateamento da tecnologia da informática tem tornado essas ferramentas cada vez mais acessíveis na educação e, em particular na educação em Ciências uma perspectiva de trabalho tem se dado por meio do desenvolvimento de ambientes de modelagem computacional.

Entretanto, a inserção dessa perspectiva demanda o delineamento de uma investigação que inclua tanto o desenvolvimento de atividades de modelagem quanto a efetiva utilização no ambiente escolar para que se possa concluir sobre as reais possibilidades de integração no cotidiano de sala de aula (RAMPINELLI e FERRACIOLI, 2006). Assim, espera-se que os resultados dessa investigação tragam novas diretrizes para a construção e elicitação de propostas para promover a evolução conceitual por meio da modelagem computacional (FERRACIOLI e SAMPAIO, 2001).

Nessa perspectiva, a investigação do desenvolvimento de atividades de modelagem em nível semiquantitativo tem se dado no contexto de cursos de graduação no estudo de fenômenos de tópicos de Ciências a partir da exploração de modelos computacionais. Esse cenário se justifica por resultados relatados na literatura (e.g. FERRACIOLI et al., 2012) de que o desenvolvimento e teste de tais atividades de modelagem nesse contexto tem contribuído para a construção desse novo perfil do ensino superior no desenvolvimento de habilidades básicas e competências específicas pelos alunos (GOMES e FERRACIOLI, 2006).

Dessa forma, este artigo relata a investigação da utilização da modelagem computacional semiquantitativa no estudo do Oscilador Harmônico Simples (OHS) a partir do desenvolvimento de atividades de modelagem computacional e teste com professores de Física cursando Pós-graduação na Universidade Federal do Espírito (UFES).

\section{Aspectos teóricos}

A utilização de modelos é imprescindível para a abordagem de fenômenos e sistemas físicos no contexto do ensino. De modo geral, um modelo é considerado como um conjunto de regras e relações que descrevem objetos, sistemas, fatos ou eventos que acontecem no mundo real ou imaginário (NORMAN, 1983).

De acordo com Forrester (1968) a mente humana é adaptada para construir e usar modelos que relacionam objetos no espaço. No entanto, no caso de representações de fenômenos mais amplos com um número maior de variáveis, tais como, os relacionados a sistemas complexos e modelos dinâmicos que representem mudanças por meio do tempo, a mente humana não é apropriadamente adequada. Nesta perspectiva, pode-se recorrer a diferentes ferramentas de modelagem como um procedimento de análise e entendimento de modelos (OGBORN, 1999).

A atividade de modelagem é considerada uma ação que leva à construção dos modelos que pode ser realizada utilizando-se materiais que vão desde papel e lápis (e.g. NOVAK e GOWIN, 1988) até as tecnologias interativas, como o computador (e.g. CAMILETTI e FERRACIOLI, 2001). Assim, a modelagem computacional pode ser 
compreendida não só na criação de modelos, mas na visualização dinâmica e obtenção de dados.

No início de utilização no contexto educacional, a modelagem computacional era realizada por meio de linguagens de programação, no entanto, com o avanço tecnológico dos computadores foi possível desenvolver softwares que permitissem aos professores e alunos construírem modelos do mundo ao redor por meio da interface gráfica. Tais softwares são denominados de ambientes de modelagem computacional (CAMILETTI e FERRACIOLI, 2002).

\section{Modelagem computacional qualitativa, semiquantitativa e quantitativa}

Muitos sistemas físicos podem ser compreendidos, inicialmente, pelas tendências de variação, e outros podem ser analisados por meio da interação entre os elementos básicos. Desta forma, Bliss (1994) classifica três diferentes formas de raciocínio que podem ser abordadas nas atividades de modelagem, são: qualitativo, semiquantitativo e quantitativo.

Segundo Camiletti e Ferracioli (2002) o raciocínio quantitativo envolve a especificação de variáveis de um sistema, os valores e as relações algébricas. Essa dimensão de raciocínio envolve o entendimento de quanto a mudança de uma variável afetará a outra como no caso do estudo de filas de supermercado: caso a população dobre, quanto esse fator afetará o tempo de espera nas filas do caixa.

Gomes (2008) enfatiza que o raciocínio qualitativo envolve a utilização de lógica ou de tomada de decisões a partir da consideração de consequências sem a necessidade de especificar tanto as variáveis quanto as relações algébricas. Como exemplo, o autor sugere pensar num mundo imaginário, onde raposas e coelhos convivem em uma relação predatória podendo acontecer vários eventos. Quando uma raposa encontra um coelho pode se alimentar ou não, pois o mesmo pode fugir. Se a raposa se alimentar, o coelho evidentemente morre, caso contrário poderá morrer de fome.

Marin (2009) diz que o raciocínio semiquantitativo envolve a descrição de fenômenos onde a direção da mudança de uma parte do sistema é conhecida mas não o tamanho do efeito desta mudança sobre as demais partes. Estas variáveis não são e usualmente não podem ser quantificadas, isto é, números e unidades não podem ser atribuídas. Porém, podem possuir certa magnitude, tais como: pequeno ou grande, muito ou pouco. Uma situação típica é caracterizada pela afirmação "quanto maior a intensidade da luz, menor é a abertura da pupila". Nessa situação não há a necessidade de quantificar a intensidade da luz e a abertura da pupila, bastando saber a relação que existe entre as tendências de variação ao longo do tempo.

O presente trabalho relata um estudo realizado utilizando-se o ambiente de modelagem computacional semiquantitativo SQRLab.

\section{A modelagem computacional e o ensino de Ciências}

A investigação sobre a utilização de recursos computacionais no contexto do ensino tem sido o foco de diversos estudos em periódicos especializados. $\mathrm{Na}$ sequência, são apresentadas algumas experiências de pesquisa sobre integração 
de ambientes computacionais ao ensino Ciências a partir da modelagem computacional quantitativa, semiquantitativa e qualitativa no contexto educacional.

Camilleti e Ferracioli (2002) investigaram a integração de ambientes de modelagem computacional ao ensino de Física, sendo realizada com alunos de Ensino Superior, por meio de atividade de conteúdo específico do sistema molamassa no ambiente de modelagem computacional semiquantitativo WLinklt, que é um ambiente de modelagem computacional com base na metáfora de ícones, para a construção e simulação de modelos. Os resultados mostram que os estudantes apresentaram habilidades para desenvolver um modelo sobre a situação proposta e relacionar o comportamento apresentado pelo modelo com o esperado, alterar o modelo e explicar o comportamento apresentado pelas variáveis.

Gomes e Ferracioli (2006) apresentaram um estudo que investigou a interação entre estudantes universitários e um ambiente de modelagem computacional qualitativo em atividades de modelagem expressiva. Nesta pesquisa os autores utilizaram o ambiente de modelagem qualitativo WorldMaker a partir do desenvolvimento da atividade de modelagem expressiva, por meio da aplicação de um curso de extensão para alunos de graduação da Universidade Federal do Espírito Santo (UFES), onde foram abordados dois tópicos, um relacionado a Física e outro a Biologia. Os resultados obtidos pelos autores mostram que os estudantes foram capazes de criar e modificar o modelo do sistema proposto a partir de próprias concepções.

Mulinari e Ferracioli (2008) apresentaram um experimento de implementação da modelagem computacional no contexto do ensino de Biologia, realizadas por alunos do curso de Ciências Biológicas, por meio do desenvolvimento de um módulo educacional sobre o crescimento celular utilizando o ambiente STELLA. Os resultados revelaram que os estudantes demonstraram aparente dificuldade em trabalhar com variáveis de modo quantitativo, uma vez que ocorreu tendência dos alunos de Ciências Biológicas apresentarem um raciocínio semiquantitativo. Os autores relataram que a modelagem computacional adequadamente estruturada por meio de atividades específicas pode levar o aluno a refletir sobre os conceitos científicos abordados e concepções. Além disso, para a efetiva integração ao ensino de Biologia demanda a adequada preparação dos estudantes e professores.

Dorneles et al. (2008) analisaram diferentes concepções e raciocínios dos alunos sobre conceitos básicos envolvidos em circuitos elétricos simples, mediante o uso de simulação e modelagem computacional, especificadamente, com o software Modellus. As atividades computacionais foram utilizadas em uma experiência didática como complemento as atividades em sala de aula e no laboratório de Física para alunos da disciplina de Física II da Universidade Federal do Rio Grande do Sul (UFRGS). Os autores concluem relatando que houve melhorias estatisticamente significativas no desempenho dos alunos que trabalharam com as atividades computacionais (grupo experimental), em comparação com alunos expostos apenas ao ensino tradicional (grupo de controle). Sob o ponto de vista qualitativo, os alunos do grupo experimental mostraram maior desenvoltura na capacidade de argumentação e maior disposição para raciocínio conceitual, em vez de meramente se valerem de fórmulas. 


\section{Contextualização do estudo}

O estudo do comportamento dinâmico de sistemas oscilatórios é importante para a compreensão tanto de fenômenos físicos correlatos, quanto do processo de construção de conhecimento em Física, pois os conceitos fundamentais necessários para o entendimento se encontram nas mais diversas áreas da Física. Dessa forma, a aprendizagem desses conceitos por parte do estudante se faz necessária desde o primeiro contato no Ensino Médio por meio do sistema massamola.

Algumas vezes os tópicos tratados em livros textos de Ciências apresentam aspectos que nem sempre possibilitam ao estudante imediata percepção da realidade. Uma abordagem tradicional estática dos fenômenos físicos não é suficiente para levar esse estudante a compreender os conceitos científicos envolvidos. Uma proposta alternativa a essa abordagem tradicional é por meio da utilização da modelagem computacional (FERRACIOLI et al., 2012), uma vez que essa é dinâmica, ou seja, o modelo construído pode ser simulado, possibilitando que o estudante visualize o sistema abordado e entenda os princípios físicos que determinam o comportamento com a evolução temporal. Assim, os resultados desse processo pode auxiliá-lo na reestruturação e melhoria do conhecimento prévio do tópico em estudo.

Entretanto, a escolha de um ambiente de modelagem computacional adequado é fundamental para atingir a perspectiva descrita. Marchewka et al. (2004) apontam que a simples inclusão da programação como alternativa de modelagem computacional pode tornar o estudo da Física obscuro, sem promover o aprofundamento desejado. Neste estudo é utilizado o ambiente de modelagem computacional SQRLab, que permite a construção de modelos com base em variáveis do sistema em estudo e, por meio de saídas gráficas, proporciona uma visualização dinâmica do comportamento das equações horárias do modelo. Nessa perspectiva de trabalho, o estudante é levado a privilegiar o enfoque nos conceitos físicos ao invés de se preocupar com as linhas de programação, tarefa típica quando da utilização de recursos computacionais.

Até o momento, com a utilização do ambiente de modelagem SQRLab foram desenvolvidos estudos sobre aquecimento global com estudantes de Ensino Médio (MARIN, 2009) e um estudo sobre sistemas oscilantes com estudantes de Ensino Superior (VERBENO et al., 2011). O primeiro estudo desenvolvido revelou que apesar de dificuldades iniciais relacionadas às definições de sistema e fenômeno de interesse, mostrou-se adequado como recurso de construção e simulação de modelos, uma vez que auxiliou os estudantes tanto na validação do modelo construído quanto na verificação da previsão de comportamento gráfico construídos no papel. No segundo estudo, foi possível observar que os estudantes foram levados a refletir sobre as próprias concepções de sistemas oscilantes e os resultados das atividades de modelagem revelaram indícios de uma evolução conceitual em direção ao conhecimento científico com relação ao tema. 
O SQRLab é um ambiente de modelagem semiquantitativa que permite a construção de modelos sobre fenômenos da natureza a partir do conhecimento das variáveis que traduzem esse fenômeno e das relações causais (MARIN, 2009). O SQRLab possui duas estruturas básicas: as variáveis que representam todas as grandezas do sistema alvo, e as ligações que estabelecem as relações entre as grandezas.

As variáveis podem receber valores de outras variáveis e tratá-los de forma diferente como uma média, soma ou um produto. As ligações podem ser de dois tipos distintos: proporcional, quando uma variável recebe o exato valor de outra, e taxa, quando uma variável tem o valor modificado a cada passo de tempo por um fator especificado por outra variável.

A Figura 1 mostra a tela principal do SQRLab ressaltando as principais funcionalidades de modelagem e simulação. Na área de modelagem é apresentada uma estrutura com duas variáveis e uma relação entre as mesmas. A janela de gráficos permite visualizar a evolução temporal do valor de quaisquer variáveis (RAMPINELLI e FERRACIOLI, 2006). Pode-se observar, que o gráfico mostra uma reta inclinada positiva, ou seja, V1 causa uma variação linear no valor de V2 e o valor de V1 é o coeficiente angular da reta.

Figura 1 - Relação causal entre a variável V1 e a variável V2, ou seja, V1 causa uma modificação em V2.

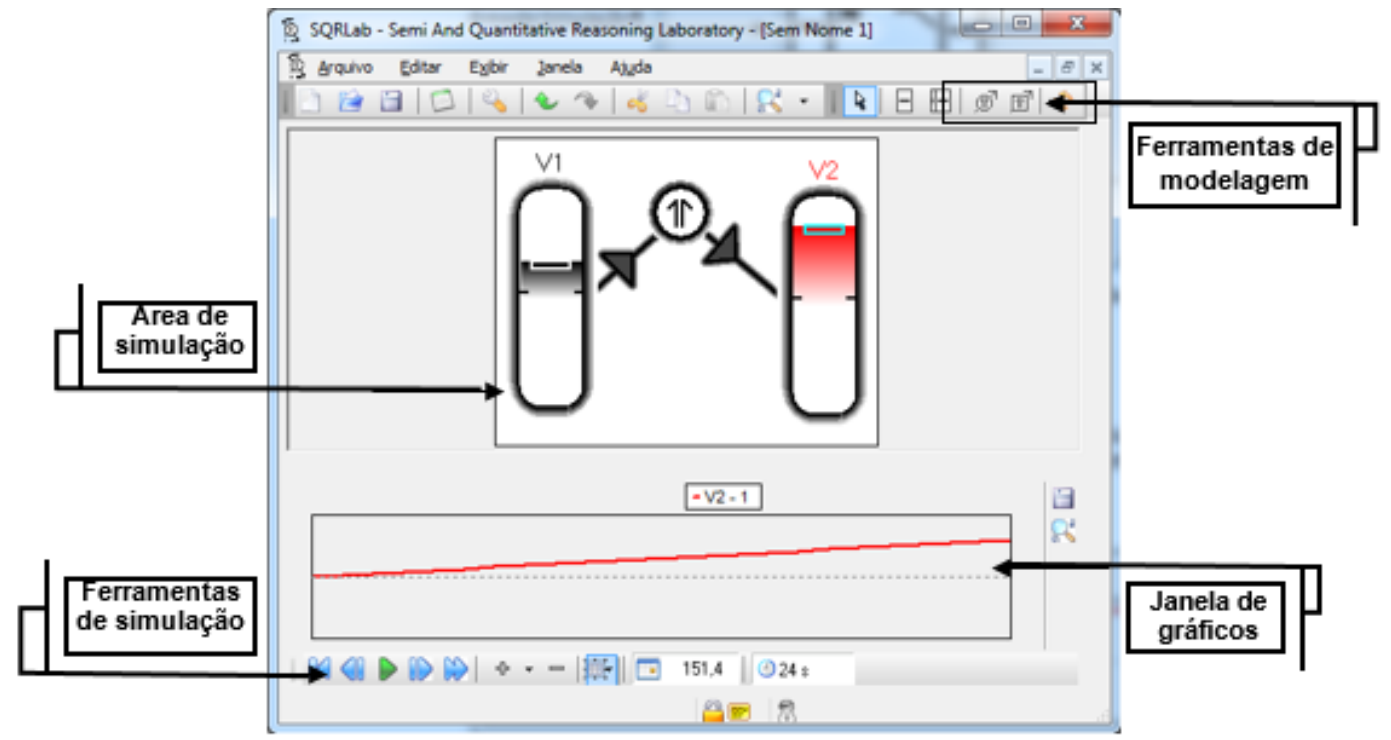

No ambiente SQRLab as relações podem ainda ser positivas, quando a causa é diretamente proporcional, ou negativas, caso contrário. Quando uma variável é definida como uma soma, todas as ligações que chegarem serão somadas, caso positivas, ou subtraídas, caso negativas. Se uma variável for definida como um produto, todas as ligações que chegarem serão multiplicadas, caso positivas, ou divididas, caso negativas.

A combinação de variáveis e relações pode fornecer o comportamento dos mais variados fenômenos e sistemas, sendo que as condições para que isso aconteça são o domínio sobre o sistema alvo a ser modelado e o domínio sobre 
como este sistema pode ser representado por meio do ambiente SQRLab. Assim, é possível construir os mais diversos modelos físicos, indo do mais simples, tal como o modelo de uma partícula em queda livre, ao mais complexo, tal como o modelo do sistema massa-mola linearmente amortecido (HICKMANN e LIBARDI, 1997).

\section{Concepção do estudo}

O presente trabalho trata do desenvolvimento de atividades de modelagem utilizando o ambiente de modelagem semiquantitativa denominado SQRLab, que foi desenvolvido pelo Laboratório de Tecnologias Interativas Aplicadas à Modelagem Cognitiva (ModeLab) da UFES. O ambiente SQRLab, disponível no endereço eletrônico <sqrlab.modelab.org>, é um software que permite a construção de modelos sobre fenômenos da natureza a partir do conhecimento das variáveis que traduzem esse fenômeno e das relações causais (FERRACIOLI et al., 2012).

O objetivo deste trabalho é investigar a integração de um módulo educacional sobre o Oscilador Harmônico Simples (OHS) com base na modelagem computacional como proposta para o ensino deste tópico no contexto de sala de aula para cursos de graduação em Ciências Exatas. Assim, um módulo educacional é estruturado em três níveis: objetivo, conteúdo instrucional e prática e avaliação (FEHSENFELD e FERRACIOLI, 2007). No contexto deste estudo, o módulo educacional desenvolvido para o experimento foi estruturado da seguinte forma: um modelo computacional desenvolvido no ambiente SQRLab com o objetivo de levar o estudante à reflexão a respeito do comportamento da amplitude e da constante de fase sujeitos as diferentes condições iniciais no sistema massa-mola, na busca de promover um melhor entendimento a respeito desses conceitos, cumprindo assim o objetivo; um roteiro de atividades que visou promover uma abordagem teórica e prática bem como produzir dados a partir das simulações computacionais para levar o estudante a responder o questionário previamente estabelecido cumprindo, assim, as etapas conteúdo instrucional e prática e avaliação. No Apêndice 1 são descritos as seis atividades na qual é estruturada o roteiro de atividades.

\section{Descrição da coleta de dados}

Para a realização desse trabalho não foi possível obter amostragem aleatória e o estudo foi desenvolvido com 10 professores de Física do Ensino Médio cursando Pós-graduação na UFES. Durante o experimento foi disponibilizado para cada professor o módulo educacional e um computador para os trabalhos no ambiente SQRLab. O experimento foi ministrado nas dependências do ModeLab que pertence ao Departamento de Física da UFES. As atividades do experimento foram realizadas por cada professor separadamente e concluídas em uma aula com duração aproximada de 2 horas. Nos primeiros 30 minutos realizou-se uma aula expositiva onde se descreveu as funcionalidades do ambiente SQRLab, em seguida, os professores foram solicitados a realizarem as atividades do módulo educacional. À medida que terminavam as atividades, os professores eram solicitados a avaliar o módulo educacional por meio de uma planilha de avaliação. 


\section{Análise da avaliação do módulo educacional}

O módulo educacional foi avaliado utilizando-se uma planilha de avaliação de módulos educacionais (MULINARI e FERRACIOLI, 2008) abordando três aspectos do material desenvolvido. Tal planilha foi desenvolvida utilizando-se uma escala Likert (CHIMI e RUSSELL, 2009), uma vez que o objetivo desta avaliação foi verificar o nível de adequação do módulo ao objetivo proposto com base na impressão dos avaliadores, que eram professores da educação básica, durante a realização do módulo. De acordo com o questionário, cada aspecto do módulo educacional poderia receber uma dentre cinco possibilidades, indo de 1 (discordo plenamente), 2 (discordo), 3 (não concordo nem discordo), 4 (concordo) e 5 (concordo plenamente). 0 número de possibilidades foi considerado adequado para o estudo em questão, pois os avaliadores foram instruídos acerca dos requisitos de um módulo educacional. Vale ressaltar que esta planilha de avaliação não tinha o objetivo de avaliar a aprendizagem dos professores promovida pelo módulo.

Os aspectos da avaliação foram: conteúdo, didático e aprendizagem. O Quadro 1 apresenta as médias dos dados coletados referente a cada um dos itens desses aspectos. Assim, observa-se que em todos os itens a maioria dos professores respondeu que concorda com as afirmações apresentadas, logo, o módulo educacional mostrou-se apropriado ao objetivo proposto, indicando a adequação para reelaborar o conhecimento sobre o tema estudado por meio do sistema massa-mola, como também, a contribuição para o desenvolvimento de habilidades de interpretação de gráficos, elaboração de hipóteses e identificação das relações entre as variáveis do sistema. Sendo necessárias apenas algumas modificações: uma revisão sobre a organização lógica das informações do conteúdo referente ao item A2; uma revisão do item $\mathrm{C} 1$ que trata dos recursos disponibilizados para a execução das tarefas, conforme sugerido por um professor; e em relação ao item C1 na qual um professor sugeriu a reformulação de uma questão com o objetivo de levar o aluno a refletir sobre alguns conceitos básicos da simulação.

Em resumo, mesmo que tenha havido sugestões para a melhoria do módulo educacional, imediatamente implementadas para a utilização, os resultados da análise dos dados revelam que a avaliação do módulo educacional foi positiva e os professores que o avaliaram o consideraram satisfatório para o objetivo a que se propõe, alguns demonstrando, também, favoráveis e otimistas a esse tipo de iniciativa com a possível extensão para o Ensino Médio. De fato, como apresentado no item da avaliação final, quando foi solicitado aos professores que atribuíssem uma nota final entre 0 e 5 ao módulo educacional, foi obtido uma média de 4.0, o que indica boa receptividade por parte desses professores quanto á utilização desse tipo de ferramenta computacional visando a promoção da aprendizagem de tópicos de Física. 
Quadro 1 - Resultados da avaliação do módulo educacional.

\begin{tabular}{|l|c|}
\hline A: Aspecto de avaliação: conteúdo & Média \\
\hline A1. As informações apresentadas são adequadas aos objetivos do módulo. & 3.8 \\
\hline A2. As informações estão organizadas de forma lógica. & 3.4 \\
\hline A3. O conteúdo é apresentado de maneira clara e objetiva. & 4.4 \\
\hline B: Aspecto de avaliação: didático & Média \\
\hline B1. O módulo é adequado ao contexto educacional da disciplina em que foi usado. & 4.2 \\
\hline B2. O módulo favorece a reflexão a respeito do conteúdo apresentado. & 4.4 \\
\hline B3. O módulo possui atividades que incentivam a formulação de hipóteses. & 3.9 \\
\hline $\begin{array}{l}\text { B4. O módulo possibilita a verificação de hipóteses por meio da análise de resultados e e } \\
\text { simulações. }\end{array}$ & 4.1 \\
\hline B5. A linguagem utilizada está de acordo com os objetivos do módulo. & 3.9 \\
\hline C: Aspecto de avaliação: aprendizagem & Média \\
\hline C1. O módulo possui recursos que motivam a aprendizagem. & 3.5 \\
\hline C2. O módulo contribui para a aprendizagem de novos conhecimentos sobre o tema. & 3.7 \\
\hline C3. O módulo o auxilia a reelaborar o conhecimento prévio sobre o tema. & 3.6 \\
\hline C4. O módulo contribui para o desenvolvimento de habilidades de: & \\
\hline a) interpretação de gráficos. & 4.3 \\
\hline b) elaboração de hipóteses. & 4.2 \\
\hline c) identificação de relações entre variáveis. & 4.2 \\
\hline D: Avaliação final & Média \\
\hline D1. Qual a nota final para o módulo? & 4.0 \\
\hline
\end{tabular}

Análise das respostas nas atividades do módulo educacional

Os dados coletados neste estudo são de natureza qualitativa e para análise qualitativa foi procedida de uma categorização de respostas seguindo, em linhas gerais, as diretrizes apresentadas por Bardin (1977). Essa avaliação teve o objetivo de analisar se o módulo educacional promovia a reflexão, por parte dos professores, a respeito dos conceitos de constante de fase, da amplitude, das condições iniciais do sistema e das equações de movimento $x(t)$ e $v(t)$ e as previsões gráficas. As respostas coletadas nas atividades $A, B, D, E$ e $F$ são analisadas nas próximas seções, comparando as questões que abordavam o mesmo conceito nessas diferentes atividades. Portanto, os Quadros 2, 3, 4 e 5 mostram as respostas dos professores para cada questão confrontando-as nos diferentes momentos das atividades do módulo. Assim, a simbologia utilizada nesses quadros tem o significado: $\mathrm{C}=$ respondeu corretamente $\mathrm{e} \mathrm{I}=$ respondeu incorretamente. 


\section{Análise dos dados da questão 1}

Nas quatro atividades A, B, E e F a questão 1 abordava o conceito de amplitude. Logo, por meio do Quadro 2 observa-se que os resultados exibidos apresentam quatro categorias de respostas. Em vermelho, o professor sete ao final das atividades justifica o mau desempenho nessa questão dizendo: "faz muito tempo que não estudo sobre o Oscilador Harmônico, não lembro de nada“. Por outro lado, ao examinar as respostas dos professores 1, 5, 6 e 9, em amarelo, observa-se que apresentaram um bom desempenho nas duas primeiras atividades $A$ e $B$, onde as condições iniciais são as mais comumente estudadas, para v_ $0=0$, porém, ao se defrontarem com uma nova situação física nas atividades seguintes onde $v \_0>0$ apresentaram as mesmas respostas nas duas atividades E e F, ambas erradas. Assim, a resposta adequada para a atividade $F$ era $A=v \_0$, no entanto, os professores responderam conforme o exemplar abaixo:

\section{$A= \pm X$}

De acordo com o excerto acima, observa-se que os professores por estudarem mais usualmente apenas situações em que v_ $0=0$ foram levados a resposta errada.

Quadro 2 - Respostas dos professores na questão 1 que aborda o conceito de amplitude.

\begin{tabular}{|c|c|c|c|c|}
\hline \multicolumn{5}{|c|}{ QUESTÃO 1 } \\
\hline & \multicolumn{4}{|c|}{ Atividades } \\
\hline \multirow{2}{*}{ PROFESSORES } & A & B & E & F \\
& Sem SQRLAB & Com SQRLAB & Com SQRLAB & Sem SQRLAB \\
\hline 1 & C & C & I & I \\
\hline 2 & C & C & I & C \\
\hline 3 & C & C & C & C \\
\hline 4 & C & C & C & C \\
\hline 5 & C & C & I & I \\
\hline 6 & C & C & I & I \\
\hline 7 & I & I & I & I \\
\hline 8 & C & C & C & C \\
\hline 9 & C & C & I & I \\
\hline 10 & C & C & C & C \\
\hline
\end{tabular}

Assim, observa-se que os quatro professores 1, 5, 6 e 9 demonstraram indícios de uma deficiência na compreensão correta do conceito de amplitude no OHS que é definido em função das condições iniciais x_0 e v_0, também, da frequência angular $\omega$, ou seja, $A=\sqrt{ }\left(x \_0^{\wedge} 2+\left(v \_0^{\wedge} 2\right) / \omega^{\wedge} 2\right)$. No entanto, o comportamento do professor 2, em verde, no sentido de reformular a resposta de maneira correta ao final das atividades, pode ser um indicativo de que as atividades pode tê-lo levado a reestruturação de concepções do senso comum, ao mesmo tempo, que aponta para a necessidade de realização de novos estudos para que possa ser conclusivo. 


\section{Análise dos dados da questão 2}

A questão 2 abordava o conceito de constante de fase nas atividades A, B, D e F. Assim, ao analisar as respostas dos professores confrontadas em cada atividade no Quadro 3 pode-se categorizar em quatro padrões de respostas.

Quadro 3 - Respostas dos professores na questão 2 que aborda o conceito de constante de fase.

\begin{tabular}{|c|c|c|c|c|}
\hline \multicolumn{5}{|c|}{ QUESTÃO 2 } \\
\hline & \multicolumn{5}{|c|}{ Atividades } \\
\hline \multirow{2}{*}{ PROFESSORES } & A & B & D \\
& Sem SQRLAB & Com SQRLAB & Com conteúdo teórico & Sem SQRLAB \\
\hline 1 & C & C & C & C \\
\hline 2 & I & I & C & C \\
\hline 3 & C & C & C & C \\
\hline 4 & C & C & C & C \\
\hline 5 & C & C & C & C \\
\hline 6 & C & C & C & C \\
\hline 7 & I & I & I & I \\
\hline 8 & I & C & C & C \\
\hline 9 & I & C & C & C \\
\hline 10 & I & I & C & I \\
\hline
\end{tabular}

Em vermelho, os professores 7 e 10, ao final das atividades, justificaram verbalmente o mau desempenho na questão 2 declarando que "A utilização do termo constante de fase nos fez procurar alguma grandeza que fosse constante e independente da fase. Nos conhecíamos essa grandeza apenas como fase inicial". Neste caso, os professores confundiram o termo "constante de fase" com a frequência angular, pois, conhecia esse conceito apenas pelo nome de fase inicial.

A reformulação das respostas por parte dos professores 2, 8 e 9, em verde, no decorrer das atividades pode ser um indicativo de que foram levados a alterar as respostas a respeito do conceito de constante de fase devido à utilização do módulo educacional, fato que ainda demanda um maior aprofundamento. Apesar dos professores 7 e 10 terem confundido o nome dado ao conceito de constante de fase, esse fato pode ser evitado utilizando os dois termos - constante de fase e fase inicial.

\section{Análise dos dados da questão 3}

A questão 3 nas atividades A, B e $F$ abordava os conceitos das funções harmônicas $x(t)$ e $v(t)$. Dessa forma, ao examinar o Quadro 4 onde as respostas dos professores das atividades A, B e F são comparadas pode-se ver 3 categorias de respostas. 
Quadro 4 - Respostas dos professores na questão 3 que aborda o conceito das funções $x(t)$ e $v(t)$.

\begin{tabular}{|c|c|c|c|}
\hline \multicolumn{4}{|c|}{ QUESTÃO 3 } \\
\hline & \multicolumn{3}{|c|}{ Atividades } \\
\hline \multirow{2}{*}{ PROFESSORES } & A & B & F \\
& Sem SQRLAB & Com SQRLAB & Sem SQRLAB \\
\hline 1 & C & C & C \\
\hline 2 & C & C & C \\
\hline 3 & C & C & C \\
\hline 4 & C & C & C \\
\hline 5 & C & C & C \\
\hline 6 & C & C & C \\
\hline 7 & I & I & I \\
\hline 8 & I & C & C \\
\hline 9 & C & C & C \\
\hline 10 & I & I & I \\
\hline
\end{tabular}

Durante a realização do módulo educacional o professor 8, em verde, se manifestou dizendo: "não lembrava das equações mas por meio do gráfico é possível analisar". Esse comentário mostra que a análise dos resultados gráficos obtidos no modelo computacional o professor 8 pôde inferir quais eram as funções que estavam associadas a esses resultados

Ao examinar as respostas dos professores 7 e 10 na questão 3 observa-se que apresentam respostas similares para todas as atividades, conforme exemplo que segue:

$$
\begin{aligned}
& x(t)=x_{0} \operatorname{sen}(\omega t) \\
& v(t)=v_{0} \cos (\omega t)
\end{aligned}
$$

As respostas corretas esperadas para que os professores apresentassem eram as seguintes:

$$
\begin{aligned}
& x(t)=x_{0} \cos (\omega t+\varphi) \\
& v(t)=-\omega x_{0} \operatorname{sen}(\omega t+\varphi)
\end{aligned}
$$

De acordo com o exemplo, percebe-se que os professores confundiram as relações entre as equações de movimento e as funções trigonométricas de seno e cosseno. Além disso, demonstraram não entender a dependência de $x(t)$ e $v(t)$ com relação a constante de fase $\phi$. Esse comportamento dos professores 7 e 10 foi verificado no decorrer das três atividades.

Portanto, os resultados da análise do professor 8 indicam que o modelo computacional pode ter contribuído para o desenvolvimento de habilidades de interpretação de gráficos. No entanto, o desempenho dos professores 7 e 10 revelam deficiência por parte desses dois professores no entendimento das equações de movimento do OHS. 


\section{Análise dos dados da questão 4}

Quadro 5 - Respostas dos professores na questão 4 com relação ao comportamento gráfico de $v(t)$ e $x(t)$

\begin{tabular}{|c|c|c|c|c|}
\hline \multicolumn{5}{|c|}{ QUESTÃO 4 } \\
\hline & \multicolumn{5}{|c|}{ ATIVIDADES } \\
\hline \multirow{2}{*}{ PROFESSORES } & A & B & E & F \\
& Sem SQRLAB & Com SQRLAB & Com SQRLAB & Sem SQRLAB \\
\hline 1 & I & I & C & C \\
\hline 2 & C & C & C & C \\
\hline 3 & C & C & C & C \\
\hline 4 & C & C & C & C \\
\hline 5 & C & C & C & C \\
\hline 6 & C & C & C & C \\
\hline 7 & C & C & C & C \\
\hline 8 & I & I & C & C \\
\hline 9 & I & I & C & C \\
\hline 10 & I & I & C & C \\
\hline
\end{tabular}

A questão 4 abordava a previsão e a comparação do comportamento gráfico de $v(t)$ e $x(t)$ nas atividades A, B, E e F. Assim, por meio do Quadro 5 observa-se que os resultados exibidos apresentam duas categorias de respostas. Em azul, os professores, de 2 a 7 encontraram resultados corretos em todas as situações e não apresentaram comentário algum. No entanto, em verde, os professores 9 e 10 apresentaram os seguintes comentários:

Professor 9: "No gráfico vxt tinha colocado vo=w.xo e é zero".

Professor 10: "Na atividade anterior acabei iniciando o gráfico com xo>0 (falta de atenção) e o gráfico da velocidade ficou invertido em relação ao gráfico da posição".

Esses comentários mostram que ao compararem as respostas da atividade $\mathrm{A}$ com a obtida no modelo perceberam aonde tinham cometido o erro. Dessa forma, o procedimento desses quatro professores de alterar a forma da previsão de comportamento gráfico para maneira correta após a interação com o modelo computacional, parece revelar que o módulo educacional mostrou suporte a esses professores para perceberem as diferenças e inconsistências entre o entendimento prévio e, o resultado obtido na simulação do SQRLab, motivandoos a refletir sobre o efeito da mudança das condições iniciais de um OHS na evolução gráfica de x(t) e v(t) do sistema.

\section{Discussão e Conclusões}

Em relação a avaliação do módulo educacional por partes dos professores, conclui-se que o modelo computacional não contém erro conceitual. Além disso, os resultados revelam também que conduziu bem o professor nas atividades, sendo necessárias apenas pequenas reformulações. Portanto, os professores mostraram-se interessados pelas atividades de modelagem como uma ferramenta 
de apoio aos processos de ensino, fato também relatado no estudo de Rampinelli e Ferracioli (2006). Com base nestes resultados, o módulo foi reformulado para uma futura aplicação com uma amostra maior.

Sobre a análise das respostas das atividades foi possível observar um progresso no entendimento dos professores ao longo das atividades de modelagem em direção ao conceito científico (GOMES e FERRACIOLI, 2006). Alguns professores que inicialmente responderam de maneira errônea, após as realizações das atividades reformularam as repostas em direção à reposta correta, com base na observação dos resultados obtidos no ambiente. Esse fato pode ser um indicativo de que as atividades de modelagem motivou-os a refletir sobre a consistência de entendimento prévio com relação aos conceitos de um OHS, corroborando com o que foi descrito no estudo de Camiletti e Ferracioli (2002).

No entanto, a análise das respostas obtidas, também, mostra que alguns professores diante uma nova situação física com diferentes condições iniciais demonstraram deficiência no conhecimento dos conceitos de amplitude e constante de fase, pois basearam as respostas nas concepções de situações físicas comumente trabalhadas nos livros de Física. Uma possível explicação para esse desempenho é que no ensino tradicional da Física, via de regra, não é explorado a influência das condições iniciais v_(0)e x_0 com relação à amplitude e a constante de fase do bloco em um OHS. Assume-se, na maioria das vezes, v_0 $0=0$, sem uma discussão adequada e sem abordar as diversas possibilidades de situações físicas que diferentes condições iniciais podem proporcionar. Essa abordagem tradicional pode levar o estudante a não adquirir o conhecimento adequado dos conceitos de constante de fase e de amplitude, podendo, em algumas situações, levar o estudante a construção de concepções alternativas em detrimento da aprendizagem do conceito científico. Dornelles et al. (2008) no estudo de circuito ressonante (RLC), também, relata sobre a compreensão conceitual inadequada de estudantes sobre o comportamento de grandezas físicas que, em nível de Física Geral, são estudadas sem a necessária ênfase nos aspectos conceituais.

Deste modo, é importante ressaltar que os resultados desse estudo representam um avanço na investigação sobre a integração de módulos educacionais utilizando ferramentas de modelagem computacional no contexto educacional, bem como reafirmam o potencial deste tipo de ferramenta no estudo de tópicos de Ciências. 


\title{
The computational modeling in Physics Teaching: an exploratory study on the Simple Harmonic Oscillator
}

\begin{abstract}
This study investigated the integration of an educational module based on computer modeling activities in the study of a specific topic of Sciences: Simple Harmonic Oscillator. The study consisted of planning, implementation, testing and evaluation of the educational module. The data collection instruments were divided into two parts: the modeling activities and the evaluation worksheet educational module. The test was conducted with high school physics teachers graduate studying at the Federal University of Espírito Santo. The evaluation of the data of the educational module demonstrated the fitness for purpose it has set, and modifications were suggested which had been implemented for future use. On the other hand, the results of modeling activities revealed that teachers could confront their prior understanding of the concepts covered in the simulation results and, in some cases, reinforce their alternative conceptions and in some cases evolved them to scientific knowledge.
\end{abstract}

KEYWORDS: Physics Teaching. Educational Module. 


\section{REFERÊNCIAS}

BARDIN, L. Análise de Conteúdo. Lisboa, v. 70, 1977.

BARRATT, C.; STROBEL, G. L. Sliding Friction and the Harmonic Oscillator. Am. J. Physics, v. 49, p. 500-501, 1981.

BLISS, J. From Mental Models to Modelling. In: MELLAR, Harvey (Org.). Learning with Artificial Worlds: Computer Based Modeling in the Curriculum. London: The Falmer Press, p. 27-32, 1994.

CAMILETTI, G. G.; FERRACIOLI, L. A utilização da modelagem computacional quantitativa no aprendizado exploratório de Física. Caderno Catarinense de Ensino, Florianópolis, v. 18, n. 2, p. 214-228, 2001.

CAMILETTI, G. G.; FERRACIOLI, L. A utilização da modelagem computacional semiquantitativa no estudo do sistema massa-mola. Revista Brasileira de Ensino de Física, São Paulo, v. 24, n. 2, p. 110-123, 2002.

CHIMI, C. J.; RUSSELL D. L. The Likert Scale: A Proposal for Improvement Using Quasi-Continuous Variables. In The Proceedings of the Information Systems Education Conference 2009, Washington DC, v. 26, 2009.

DORNELES, P. F. T.; ARAUJO, I. S.; VEIT, E. A.; Simulação e Modelagem Computacionais no auxílio a Aprendizagem Significativa de Conceitos Básicos de Eletricidade, Parte II - Circuitos RLC. Revista Brasileira de Ensino de Física, São Paulo, v. 30, n. 3, 2008.

FEHSENFELD, K. M.; FERRACIOLI, L. Desenvolvimento, utilização e avaliação de um módulo educacional baseado na modelagem computacional no contexto da sala de aula: um estudo sobre a primeira e a segunda leis de Newton. In: Encontro Nacional de Pesquisa em Educação em Ciência, Florianópolis, v. 6, 2007.

FERRACIOLI, L.; SAMPAIO, F. F. Informação, Ciência, Tecnologia e Inovação Curricular em Cursos de Licenciatura. Revista Brasileira de Informática na Educação, v. 8, n. 1, p. 77-85. 2001.

FERRACIOLI, L. Perspectivas e Resultados da Aprendizagem Exploratória em 
Representações e Modelagem no Processo de Ensino-Aprendizagem, Vitória, 2003.

FERRACIOLI, L.; GOMES, T. MARQUES, R.; MULINARI, M. H.; OLIVEIRA, R. R.; CAMILETTI, G. G.; MORELATO, F.; FEHSENFELD, K.; VERBENO, C. H. Ambientes de Modelagem Computacional no Aprendizado Exploratório de Física. Caderno Brasileiro de Ensino de Física, v. 29, n. 2, p. 679-707, 2012.

FORRESTER, J. Principles of Systems. Cambridge, Ma: Wright- Allen Press, 1968.

GOMES, T.; FERRACIOLI, L. A investigação da construção de modelos no estudo de um tópico de Física utilizando um ambiente de modelagem computacional qualitativo. Revista Brasileira de Ensino de Física, v. 28, n. 4, p. 453-461, 2006.

GOMES, T. A Modelagem Computacional Qualitativa Através do Ambiente ModeLab: Um Estudo Exploratório com Estudantes Universitários Desenvolvendo Atividades de Modelagem Expressiva Sobre Tópicos de Ciências. 2008. 233 f. Tese (Doutorado em Física) - Universidade Federal do Espírito Santo, Vitória, 2008.

HICKMANN, J. S.; LIBARDI, H. O Oscilador Linearmente Amortecido. Revista Brasileira de Ensino de Física, v. 19, n. 13, p. 359-363, 1997.

MARCHEWKAA, A.; ABBOTT, D. S.; BEICHNER, R. J. Oscillator Damped by a Constant-Magnitude Friction Force. Am. J. Physics, v. 72, n. 4, p. 477-483, 2004.

MARIN, F. C. M. A Utilização de Diagramas Causais no Desenvolvimento de Atividades de Modelagem Semiquantitativa: Um Estudo Exploratório com Estudantes do Ensino Médio. 220f. Dissertação (Mestrado em Física) Universidade Federal do Espírito Santo, Vitória, 2009.

MULINARI, M. H.; FERRACIOLI, L. A Utilização da Tecnologia da Informação no Ensino de Biologia: Um Experimento com um Ambiente de Modelagem Computacional. Revista Brasileira de Ensino de Ciência e Tecnologia, v. 1, p. 98115, 2008.

NORMAN, D. A. Some Observations on Mental Models. In: Gentner, Dedreand Stevens, Albert L. Mental Models. Lawrence Erlbaum Associates. 1983.

NOVAK, J.; GOWIN, D. B. Learning How to Learning. Cambridge, Ma: Cambridge 
NUSSENZVEIG, H. M., Curso de Física Básica, São Paulo: Edgard Blücher, v. 2/4, 2002.

OGBORN, J. Modeling Clay for Thinking and Learning. In Feurzeig, Wallace; Roberts, Nancy (Eds.) Modeling and Simulation in Science and Mathematics Education. Springer-Verlag, New York, p. 5-37, 1999.

RAMPINELLI, M.; FERRACIOLI, L. A Integração de um Ambiente de Modelagem Computacional Quantitativo no Estudo do Fenômeno de Colisões. Caderno Brasileiro de Ensino de Física, Florianópolis, v. 23, n. 1, p. 93-122, 2006.

VERBENO, C. H.; GOMES, T.; SILVA, R. M. A.; FERRACIOLI, L. Investigação sobre Integração de um Módulo Educacional sobre o Sistema Massa-Mola utilizando o Ambiente de Modelagem Computacional SQRLab. In: Anais do XIX Simpósio Nacional de Ensino de Física. Manaus, Amazônia, 2011.

Recebido: 22 mar. 2015

Aprovado: 26 jun. 2016

DOI: http://dx.doi.org/10.3895/rbect.v9n2.2833

Como citar: VERBENO, C. H. S.; SILVA, R. M. A.; MAZIERO, R.; GOMES, T. S.; FERRACIOLI, L. A Modelagem Computacional no Ensino de Física: Um Estudo Exploratório sobre o Oscilador Harmônico Simples. Revista Brasileira de Ensino de Ciência e Tecnologia, v. 9, n. 2, 2016. Disponível em: <https://periodicos.utfpr.edu.br/rbect/article/view/2833>. Acesso em: xxx.

\section{Correspondência:}

Carlos Henrique Santos Verbeno

Av. Fernando Ferrari, Campus de Goiabeiras, Vitória, ES, Prédio IC1, sala 7,

Goiabeiras, 29075-910 - Vitoria, ES - Brasil Direito autoral: Este artigo está licenciado sob os termos da Licença Creative Commons-Atribuição 4.0 Internacional. 
Apêndice 1

Quadro 6 - Descrição das atividades do módulo educacional.

\section{- Atividade A}

Dadas as condições iniciais do sistema massa-mola o aluno é solicitado a responder 4 questões com base nas próprias ideias sobre conceitos relacionados a este tópico, sem o auxílio do ambiente de modelagem:

1) Qual é a amplitude do movimento do bloco?

2) Qual é a constante de fase do movimento do bloco?

3) Escreva a equação da posição $x(t)$ e a equação da ve locidade v(t).

4) Esboce os gráficos: $x(t)$ posição do bloco em função do tempo e v(t) velocidade do bloco em função do tempo.

\section{- Atividade B}

É solicitado ao aluno que introduza no modelo computacional as condições iniciais do sistema apresentadas na atividade $\mathrm{A}$ e inicie a simulação do modelo. Em seguida, com o auxílio do ambiente de mode lagem é solicitado ao estudante que respond a as seguintes questões:

1) Qual é a amplitude do movimento do bloco?

2) Qual é a constante de fase do movimento do bloco?

3) Escreva a equação da posição $\mathrm{x}(\mathrm{t})$ e a equação da posição $\mathrm{v}(\mathrm{t})$ que representam os gráficos obtidos na simulação.

4) As saídas gráficas obt idas na simulação são seme lhantes ao esboço traçado anteriormente? Explique a diferença.

\section{- Atividade C}

Consiste em uma abordagem teórica do sistema massa-mola, a qual descreve os conceitos re lacionados a este tópico e enfatiza a relação entre as condições in icia is do sistema e a constante de fase.

\section{- Atividade D}

Conforme discutido na atividade $\mathrm{C}$ é solicitado ao aluno que responda uma questão que aborda a re lação entre as condições iniciais e a constante de fase:

1) Calcule as condições iniciais do sistema para uma constante de fase $\varphi=\pi / 4$, adotando o valor de $\omega_{0}=1$, ou seja, $\mathrm{m}=\mathrm{k}$.

\section{- Atividade $\mathbf{E}$}

É solicitado ao aluno introduzir as características do sistema encontradas na atividade De com o auxílio do ambiente de modelagem responder as questões abaixo:

1) Qual é a amplitude do movimento do bloco?

2) O gráfico encontrado no ambiente de modelagem corresponde ao previsto pela Figura 1 na abordagem teórica da atividade C? Explique a diferença.

\section{- Atividade F}

Sem a utilização do mode lo, é solicitado ao aluno que responda as mesmas questões da atividade A no entanto, com condições inicia is dife rentes.

1) Qual é a amplitude do movimento do bloco?

2) Qual é a constante de fase do movimento do bloco?

3) Escreva a equação da posição $x(t)$ e a equação da ve locidade $v(t)$.

4) Esboce os gráficos: $x(t)$ posição do bloco em função do tempo e v(t) velocidade do bloco em função do tempo. 